\title{
SIRT3 in Cardiac Physiology and Disease
}

\author{
Christoph Koentges, Christoph Bode and Heiko Bugger* \\ Division of Cardiology and Angiology I, Heart Center Freiburg University, Freiburg, Germany
}

Functional defects in mitochondrial biology causally contribute to various human diseases, including cardiovascular disease. Impairment in oxidative phosphorylation, mitochondrial oxidative stress, and increased opening of the mitochondrial permeability transition pore add to the underlying mechanisms of heart failure or myocardial ischemia-reperfusion (IR) injury. Recent evidence demonstrated that the mitochondrial NAD+-dependent deacetylase sirtuin 3 (SIRT3) may regulate these mitochondrial functions by reversible protein lysine deacetylation. Loss of function studies demonstrated a role of impaired SIRT3 activity in the pathogenesis of myocardial IR injury as well as in the development of cardiac hypertrophy and the transition into heart failure. Gain of function studies and treatment approaches increasing mitochondrial $\mathrm{NAD}^{+}$availability that ameliorate these cardiac pathologies have led to the proposal that activation of SIRT3 may represent a

OPEN ACCESS

Edited by:

Manfredi Tesauro,

University of Rome Tor Vergata, Italy

Reviewed by:

Jeonga Kim,

University of Alabama at Birmingham,

USA

Petra Kienesberger,

Dalhousie University, Canada

*Correspondence: Heiko Bugger heiko.bugger@universitaetsherzzentrum.de

Specialty section:

This article was submitted to

Cardiovascular Metabolism,

a section of the journal

Frontiers in Cardiovascular Medicine

Received: 08 August 2016 Accepted: 30 September 2016

Published: 13 October 2016

Citation:

Koentges C, Bode $C$ and Bugger $H$ (2016) SIRT3 in Cardiac

Physiology and Disease.

Front. Cardiovasc. Med. 3:38. doi: $10.3389 /$ fcrm.2016.00038 promising therapeutic strategy to improve mitochondrial derangements in various cardiac pathologies. In the current review, we will present and discuss the available literature on the role of SIRT3 in cardiac physiology and disease.

Keywords: sirtuin 3, mitochondria, heart failure, ischemia-reperfusion injury

\section{INTRODUCTION}

The sirtuin family of protein deacetylases consists of seven mammalian members (SIRT1-7), which are orthologs of the silent information regulator two (Sir2) that was first identified in yeast almost two decades ago $(1,2)$. Sirtuins are classified as class III histone deacetylases, requiring $\mathrm{NAD}^{+}$as a cosubstrate, which acts as an acceptor molecule for the acetyl group removed from the target protein (3). The need of $\mathrm{NAD}^{+}$as a cosubstrate links activity of sirtuins tightly to the cellular energy status making them potential redox sensors (4-6). SIRT3, SIRT4, and SIRT5 are primarily localized inside the mitochondria, and SIRT3 possesses the most robust deacetylase activity of all mitochondrial sirtuins $(7,8)$. Although named histone deacetylases, numerous non-histone protein targets have been identified using large-scale proteomic analyses, also including proteins localized outside of mitochondria $(9,10)$. Deacetylation targets of SIRT3 include metabolic enzymes involved in fatty acid oxidation (FAO) and glucose oxidation (GLOX), protein subunits of the electron transport chain (ETC), as well as enzymes involved in oxidative stress defense and mitochondrial integrity $(7,10-21)$.

SIRT3 is highly expressed in tissues with high metabolic turnover and mitochondrial content, including the heart and its role in cardiac physiology and pathology have been increasingly investigated in recent years $(8,17,22-29)$. Under normal physiologic conditions, about $95 \%$ of ATP used to maintain cardiac pump function is regenerated within mitochondria by the oxidation of fatty acids and glucose, with some contribution from other substrates as well (30). Mitochondrial derangements such as defects in substrate oxidation and oxidative phosphorylation, mitochondrial 
oxidative stress, and increased sensitivity for opening of the mitochondrial permeability transition pore $(\mathrm{mPTP})$ are major mechanisms underlying clinically important cardiac pathologies, such as heart failure and ischemia-reperfusion (IR) injury (31-36). A number of studies provided evidence that SIRT3 regulates these mechanisms in the heart. In this review, we will present and discuss the current knowledge on the role of SIRT3 in cardiac physiology and disease and potential therapeutic strategies aiming at modulating SIRT3 activity.

\section{SIRT3 IN CARDIAC PHYSIOLOGY}

\section{SIRT3 and Cardiac Function}

The impact of SIRT3 in the regulation of cardiac function has been evaluated in a number of studies using mice with global deletion of SIRT3 (SIRT3 ${ }^{-1-}$ ) $(13,17,22-26,29)$. Using echocardiography, fractional shortening (FS) and ejection fraction (EF) were not different between SIRT3 ${ }^{-1-}$ and wild-type (WT) mice at 8 weeks of age $(17,23,25,28,29,37)$. However, in older animals (24 weeks of age), SIRT ${ }^{-1-}$ mice displayed a decrease in EF and FS, accompanied by increased systolic and diastolic diameters and volumes of the left ventricle (LV) indicative of $\operatorname{LV}$ dilation $(23,28)$. Thus, SIRT3 $^{-1-}$ mice seem to develop an accelerated age-related decline in cardiac contractile function $(23,28)$. Interestingly, when perfused in the isolated working heart model, even 8-week-old SIRT3 $^{-1-}$ mice display contractile defects, such as a decrease in cardiac power, cardiac output, and developed pressure $(22,23)$. Possibly, contractile dysfunction could not be observed in young mice in vivo but only ex vivo since neurohormonal compensatory mechanisms may only be present in the live animal (e.g., reninangiotensin-aldosterone-system, autonomic nervous system, changes in vasomotor and/or heart rate), but absent in isolated perfused hearts. With increasing age, the progress in cardiac remodeling due to SIRT3 deficiency (e.g., increased fibrosis, LV dilation) may dominate these compensatory mechanisms leading to obvious cardiac dysfunction detectable by echocardiography. Furthermore, aging-associated abnormalities in mitochondrial function, such as increased oxidative stress, mutations of mitochondrial DNA, and decreased mitochondrial respiration may predispose for a stronger impact of SIRT3 deficiency on overall mitochondrial function and integrity in older versus younger mice (38). Interestingly, one study did not observe impairment in contractile function in 24- to 26-week-old mice in isolated working hearts (24). In this study though, hearts were perfused in the presence of insulin and higher concentrations of fatty acids compared to Koentges and colleagues (23). The impact of insulin and substrate availability on cardiac function and energetics in SIRT3 $^{-1-}$ mice remains to be determined in more detail.

Further evidence supporting the proposal of SIRT3 being required to maintain cardiac function comes from studies that increased cardiac energy demand by increasing cardiac workload. Following transverse aortic constriction (TAC), SIRT3 ${ }^{-/}$mice display an accelerated development of contractile dysfunction with a more pronounced decrease in $\mathrm{EF}$ and a more pronounced increase in endsystolic volume (23). Under these conditions, SIRT3 $^{-/}$mice develop a greater degree of cardiac fibrosis and a more pronounced increase in cardiomyocyte hypertrophy
$(13,17,23,25)$. Vice versa, Sundaresan et al. could prevent cardiac contractile dysfunction and attenuate the extent of fibrosis after angiotensin II (AngII) infusion by cardiomyocyte-specific overexpression of SIRT3 (17). Interestingly, TAC-induced contractile dysfunction with concomitant reduction of SIRT3 expression in WT mice was rescued by reinduction of SIRT3 expression by administration of honokiol, a compound derived from magnolian trees (39). Taken together, these studies suggest that SIRT3 seems to be required to maintain cardiac contractile function.

\section{Targets and Pathways Regulated by SIRT3}

Cardiac function is tightly linked to the continuous delivery of energy-rich phosphates, 95\% of which are generated by mitochondrial oxidative phosphorylation. Quite a number of studies reported that SIRT3 regulates energy metabolism in various tissues, including the heart. Hirschey et al. reported decreased rates of palmitate oxidation in cardiac tissue extracts from SIRT3 ${ }^{-1-}$ mice, and Ahn et al. reported lower cardiac ATP levels in these mice $(16,40)$. These findings were confirmed in isolated working heart experiments showing decreased rates of palmitate and GLOX and lower rates of myocardial $\mathrm{O}_{2}$ consumption in SIRT3 ${ }^{-/-}$mice (23). In addition, isolated cardiac mitochondria showed lower rates of ATP production, resulting in lower ATP/AMP ratios, and thus decreased cellular energy charge (23). Impairment in mitochondrial function may be the consequence of hyperacetylation of several SIRT3 target enzymes in the absence of SIRT3. SIRT3 target enzymes range through almost all substrate oxidation and ATP-generating processes from pyruvate import (mitochondrial pyruvate carrier), TCA cycle activity and its anaplerosis (acetylCoA synthetase, aconitase, glutamate dehydrogenase, isocitrate dehydrogenase), $\beta$-oxidation of fatty acids (long-chain acyl-CoA dehydrogenase), to oxidative phosphorylation (Ndufa9, succinate dehydrogenase and ATP synthetase subunit a) and ATP translocation (adenine nucleotide translocase) $(7,11,12,14,16,19,20$, $23,40,41)$. Most of the described targets of SIRT3 are thought to be activated by SIRT3-mediated protein deacetylation.

Since SIRT3 activity is directly linked to the $\mathrm{NAD}^{+} / \mathrm{NADH}$ ratio, SIRT3 may serve as a metabolic sensor. In situations of high energy demand, the $\mathrm{NAD}^{+} / \mathrm{NADH}$ ratio rises and subsequently increases SIRT3 activity, which may translate into deacetylation of metabolic enzymes and thus acceleration of metabolic pathway flux and energy production. A dysbalance between energy demand and supply may occur during physical exercise or changes in blood pressure or during the 24-h fasting and feeding cycle, when $\mathrm{NAD}^{+}$levels oscillate in a circadian fashion (42). Given the fact that SIRT3 drives mitochondrial substrate oxidation and thus ETC flux, it is not surprising that SIRT3 participates in the detoxification of reactive oxygen species (ROS). Manganese superoxide dismutase (MnSOD), a major intramitochondrial antioxidant enzyme, has been reported to be a deacetylation target of SIRT3, and MnSOD expression is also controlled by SIRT3-mediated nuclear translocation of the transcription factor forkhead-boxprotein 3a (Foxo3a) $(17,43,44)$. SIRT3 may, thereby, protect the heart from oxidative damage induced by increased production of ROS, which may result as byproducts of accelerated ETC flux.

Of note, the assumption that a lower degree of lysine acetylation is connected to a higher activity of the affected enzyme 
should be drawn carefully. Although most of the described deacetylation targets of SIRT3 are activated by deacetylation, Fernandes et al. recently reported that increased acetylation of aconitase 2 (ACO2) increases ACO2 enzymatic activity (12). The authors argue that lower expression of SIRT3 induced by high-fat diet feeding may increase ACO2 activity, which may increase cytosolic citrate levels and subsequently inhibit acetylCoA-carboxylase, resulting in lower malonyl-CoA levels and subsequent derepression of carnitine-palmitoyl transferase (12). Lower SIRT3 levels would, thereby, adapt mitochondrial $\beta$-oxidation to higher levels of circulating fatty acids. In another study, Alrob et al. reported decreased SIRT3 protein levels after feeding a high-fat diet, which was associated with higher acetylation levels of long-chain acyl-CoA dehydrogenase (LCAD) and 3-hydroxyacyl-CoA dehydrogenase leading to higher enzyme activity and FAO rates (24). Of interest, increased acetylation of LCAD has been reported to both increase and decrease its activity. One possible explanation for these conflicting results may be differences in the acetylation pattern of LCAD, which may differentially affect enzymatic activity. For example, p53 possesses 13 potential acetylation sites in different domains (45). Mathematically, 6,227,020,800 different combinations of lysine acetylations would be possible, which may change p53 protein function. To date, only few data are available on specific acetylation patterns induced by SIRT3 or SIRT3 deficiency and the effect on target enzyme activity.

\section{SIRT3 Expression in the Heart}

SIRT3 is highly expressed in organs with high metabolic turnover, e.g., heart, liver, brain, and kidney (40). In the heart, SIRT3 expression responds to changes in energy demand (16, 23, 25, 27, $29,39,46)$. In mice, myocardial SIRT3 expression was increased following TAC or infusion of isoproterenol or AngII, probably to match oxidative capacity to increased demand for ATP regeneration in hypertrophied hearts $(17,23)$. In contrast though, when hypertrophy progresses to heart failure, or in doxorubicininduced heart failure, expression of SIRT3 was decreased $(25,27$, 47). Similarly, SIRT3 expression was decreased in human failing hearts $(48,49)$. Thus, the downregulation of SIRT3 may rather be maladaptive in the failing heart and may exaggerate mitochondrial dysfunction and oxidative stress.

A downregulation of SIRT3 was also observed in hearts of mice fed a high-fat diet, in obese, and type 2 diabetic $d b / d b$ mice, and in rodents with streptozotocin-induced type 1 diabetes, suggesting that downregulation of SIRT3 in the heart may also be a hallmark of the metabolic syndrome and diabetes mellitus, although a downregulation of SIRT3 was not observed in every single study $(24,28,50-52)$. Similar to failing hearts, SIRT3 downregulation may also contribute to myocardial mitochondrial dysfunction and oxidative stress in diabetes, but may also contribute to reduced capillary density in diabetic hearts $(28,50,53)$.

Upstream signaling that regulates SIRT3 expression is poorly understood, but may involve the master regulator of mitochondrial biogenesis and gene expression, peroxisome proliferator-activated receptor gamma coactivator $1 \alpha(\mathrm{PGC}-1 \alpha)$. Kong et al. demonstrated that PGC- $1 \alpha$ regulates SIRT3 expression via binding of ERR $\alpha$ to an ERR response element on the
SIRT3 promoter in C2C12 myotubes (54). In skeletal muscle, dihydromyricetin improved insulin sensitivity by induction of autophagy via activation of the AMPK-PGC- $1 \alpha$-Sirt3 signaling pathway (55). Thus, SIRT3 expression may be regulated by PGC$1 \alpha$, and reduced expression of SIRT3 in heart failure may thus be related to downregulation of PGC- $1 \alpha$ in failing hearts $(48,49,56)$. This hypothesis, however, remains to be tested experimentally.

\section{SIRT3 IN CARDIAC DISEASE}

\section{SIRT3 in Hypertrophy and Heart Failure}

Increasing evidence suggests a causative role of impaired SIRT3 activity in a number of cardiac pathologies, including cardiac hypertrophy and heart failure. SIRT3 ${ }^{-1-}$ mice spontaneously develop cardiac hypertrophy with increasing age $(13,17,23,29)$. The hypertrophic response to pressure overload (induced by TAC), treatment with AngII, or infusion of isoproterenol is more pronounced in $\mathrm{SIRT3}^{-1-}$ mice compared with non-transgenic controls $(13,17,23,29)$. The exacerbated hypertrophy is accompanied by a more pronounced increase in interstitial fibrosis as well. Conversely, overexpression or pharmacological activation of SIRT3 was able to ameliorate or even block cardiac hypertrophy and interstitial fibrosis in response to pressure overload or hypertrophy agonist treatment $(17,29,47)$.

Several mechanisms may contribute to the beneficial and deleterious effects of SIRT3 activation and deletion, respectively. SIRT3 has numerous targets in mitochondrial energy metabolic pathways, which are hyperacetylated in mice lacking SIRT3. A coordinated slowing of FAO, GLOX, the TCA cycle, and oxidative phosphorylation with subsequent myocardial energy depletion due to decreased SIRT3 activity may, thus, be a plausible mechanism contributing to contractile dysfunction and impaired adaptation to increased energy demands $(23,40)$. Another important mechanism may be an imbalance between ROS production and expression and activity of antioxidative enzymes, resulting in oxidative stress. SIRT3 ${ }^{-/-}$mice show signs of increased oxidative stress, e.g., increased levels of 4-HNE and TBARS $(15,23)$. Vice versa, overexpression of SIRT3 prevents ROS accumulation during hypertrophy, probably by preventing a drop in catalase and MnSOD expression after AngII infusion, likely due to an increase in FOXO3a signaling (17). In addition, SIRT3 is capable of deacetylating MnSOD, thereby increasing its activity and attenuating oxidative stress (17). Protection from oxidative stress may also attenuate activation of the ROSsensitive MAPK/ERK and PI3K/AKT signaling pathways, which are known to play a major role in the development of cardiac hypertrophy (57). Cytosolic ROS may be of minor importance though, since ROS scavenging by treatment with the antioxidant 4-hydroxy-2,2,6,6-tetramethylpiperidin-1-oxyl (TEMPOL) did not improve contractile deficits in hearts of SIRT3 ${ }^{-1-}$ mice (23). Increased fibrosis in aged SIRT $3^{-1-}$ mice and following TAC may be related to a disinhibition of transforming growth factor $\beta 1$ (TGF- $\beta 1$ ) signaling and hyperacetylation of glycogen synthase kinase $3 \beta$ (GSK3 $\beta$ ) by SIRT3 deficiency, resulting in increased expression of profibrotic genes (58).

The above mentioned mechanisms may well contribute to mitochondrial dysfunction typically observed in failing hearts. 
Indeed, SIRT3 expression is reduced and global mitochondrial protein lysine acetylation is increased in rodent models of heart failure, suggestive of impaired SIRT3 activity (48). Reduced SIRT3 expression may be the consequence of PGC-1 $\alpha$ downregulation, suggesting that impaired PGC- $1 \alpha$-SIRT3 signaling may impair mitochondrial function by several mechanisms, including inhibition of substrate oxidation due to enzyme hyperacetylation, increased oxidative stress, and impaired OXPHOS expression due to impaired PGC-1 $\alpha$ signaling per se. Another mechanism which may impair SIRT3 activity in failing hearts may be NAD ${ }^{+}$ depletion or a decrease in the $\mathrm{NAD}^{+} / \mathrm{NADH}$ ratio. Poly(ADPribose)-polymerase 1 (PARP-1) is a DNA repair enzyme which is overactivated in failing hearts, and PARP- 1 also uses $\mathrm{NAD}^{+}$as a cosubstrate (59). Overactivation of PARP-1 leads to cellular NAD ${ }^{+}$ depletion, potentially resulting in impaired SIRT3 activity (59, $60)$. A decrease in the $\mathrm{NAD}^{+} / \mathrm{NADH}$ ratio may also be caused by preexisting mitochondrial dysfunction per se. Karamanlidis et al. demonstrated a decrease in the $\mathrm{NAD}^{+} / \mathrm{NADH}$ ratio in a mouse model with complex I (Ndufs4 subunit) deficiency, resulting in heart failure (5). Since protein levels of SIRT3/4/5 were unchanged, they proposed that inhibition of SIRT3 activity by a low $\mathrm{NAD}^{+}$/ $\mathrm{NADH}$ ratio may have caused hyperacetylation of mitochondrial proteins, which would further impair the preexisting mitochondrial dysfunction due to complex I deficiency. Indeed, restoration of the $\mathrm{NAD}^{+} / \mathrm{NADH}$ ratio by treatment with $\beta$-nicotinamide mononucleotide (NMN) decreased mitochondrial protein acetylation in mice with complex I deficiency (5). Thus, both, reduced expression of SIRT3 and a decrease in $\mathrm{NAD}^{+}$availability may contribute to impaired SIRT3 activity in heart failure.

\section{SIRT3 in IR Injury}

Mitochondria play a pivotal role in the pathogenesis of myocardial IR injury. Impaired mitochondrial respiration, mitochondrial oxidative stress, and increased opening of the MPTP determine myocardial infarct size and recovery of contractile function following an ischemic episode $(61,62)$. Porter et al. reported pronounced impairment in recovery of contractile function and an increase in myocardial infarct size following ischemia in 28-week-old SIRT3 ${ }^{+/}$mice compared with WT controls (26). Regarding the mechanisms, SIRT3 ${ }^{+/-}$mice showed a decrease in complex I activity, suggesting impairment in mitochondrial function, and a decrease in MnSOD activity, suggesting impaired oxidative stress defense (26). In addition, Hafner et al. reported increased sensitivity for MPTP opening in SIRT3 ${ }^{-1-}$ mice, likely due to deacetylation of lysine 166 of cyclophilin D, a critical component of the mPTP (13). Since mitochondrial $\mathrm{NAD}^{+}$levels drop during myocardial IR in WT mice, SIRT3 activity may be impaired during IR and, thus, contribute to IR injury in the heart (63).

Of interest and in contrast to SIRT3 heterozygous mice, $\mathrm{SIRT}^{-/-}$mice showed no additional impairment in recovery of contractile function following global no-flow ischemia in the isolated working heart model (22). In addition, rates of mitochondrial $\mathrm{O}_{2}$ consumption, 4-HNE levels, and $\mathrm{Ca}^{2+}$-induced mitochondrial swelling were not different to WT controls undergoing IR (22). However, SIRT3 ${ }^{-1-}$ mice were younger (8 weeks of age) compared with 6-month-old mice in the study by Porter and colleagues (26). Thus, these results suggest an age-dependent increase in susceptibility for IR injury in SIRT3 ${ }^{-1}$ mice. In fact, the extent of IR injury generally increases with age, accompanied by abnormalities of mitochondrial biology, such as increased ROS production, decreased mitochondrial cristae density, and impairment in oxidative phosphorylation and ETC complex activities (64-71). Thus, it is tempting to speculate that lack of SIRT3 per se may not necessarily aggravate IR injury, but with increasing age, the presence of additional defects in mitochondrial function may ultimately increase the overall vulnerability of SIRT3 ${ }^{-/-}$mice for cardiac damage following myocardial IR. Of note, it has been reported that the expression of SIRT3 decreases with increasing age in various organs in mice (kidney, brain, aortic valve tissue), and that $\mathrm{NAD}^{+}$levels also drop with increasing age in $C$. elegans and liver and skeletal muscle tissue in mice $(37,72-76)$. Thus, a combination of decreased SIRT3 expression and activity may contribute to a greater extent of IR injury with increasing age.

\section{SIRT3 AS A POTENTIAL THERAPEUTIC TARGET}

Based on the evidence presented in the previous paragraphs, impaired SIRT3 activity may play an important role in the pathogenesis of cardiac hypertrophy, heart failure, and IR injury. Thus, different pharmacological approaches have been pursued to either increase SIRT3 expression or to increase SIRT3 activity by raising $\mathrm{NAD}^{+}$levels (Figure 1). Honokiol is a polyphenol that belongs to a class of neolignan biphenols and that has a high bioavailability. It is derived from bark of magnolian trees and has been widely used in traditional medicine in China, Korea, and Japan. Honokiol may exert a large number of effects, including anti-inflammatory effects, anti-apoptotic effects, antioxidant effects, anti-tumorigenic effects, may activate PPAR $\gamma$ signaling, and interferes with MAPK and PI3K/Akt signaling, among several other effects $(77,78)$. Pillai et al. recently showed that the administration of honokiol is capable of reversing cardiac hypertrophy and of rescuing contractile dysfunction following TAC in mice (39). The authors showed that honokiol was present within mitochondria and enhanced SIRT3 expression nearly two-fold, accompanied by reduced deacetylation of mitochondrial SIRT3 target proteins (39). Another natural biphenol that is contained in the skin of grapes, blueberries, and raspberries and that may modulate SIRT3 activity, is resveratrol (RSV). RSV exerts multiple effects on the cardiomyocyte, including AMPK activation, induction of autophagy, inhibition of ROS production, vasorelaxation, inhibition of atherosclerosis, antiinflammatory effects, and boosting mitochondrial biogenesis, using many different signaling pathways outlined in more detail elsewhere $(79,80)$. Regarding sirtuins, RSV was originally reported to activate SIRT1 and is thought to decrease the risk of heart disease (79). Chen et al. recently reported though that RSV prevented cardiac hypertrophy in response to hypertrophic stimuli in WT mice, and this protective effect was not observed in SIRT3 knockout mice (81). In addition, the activation of SIRT3 by RSV attenuated collagen deposition and improved cardiac contractile function (81). 


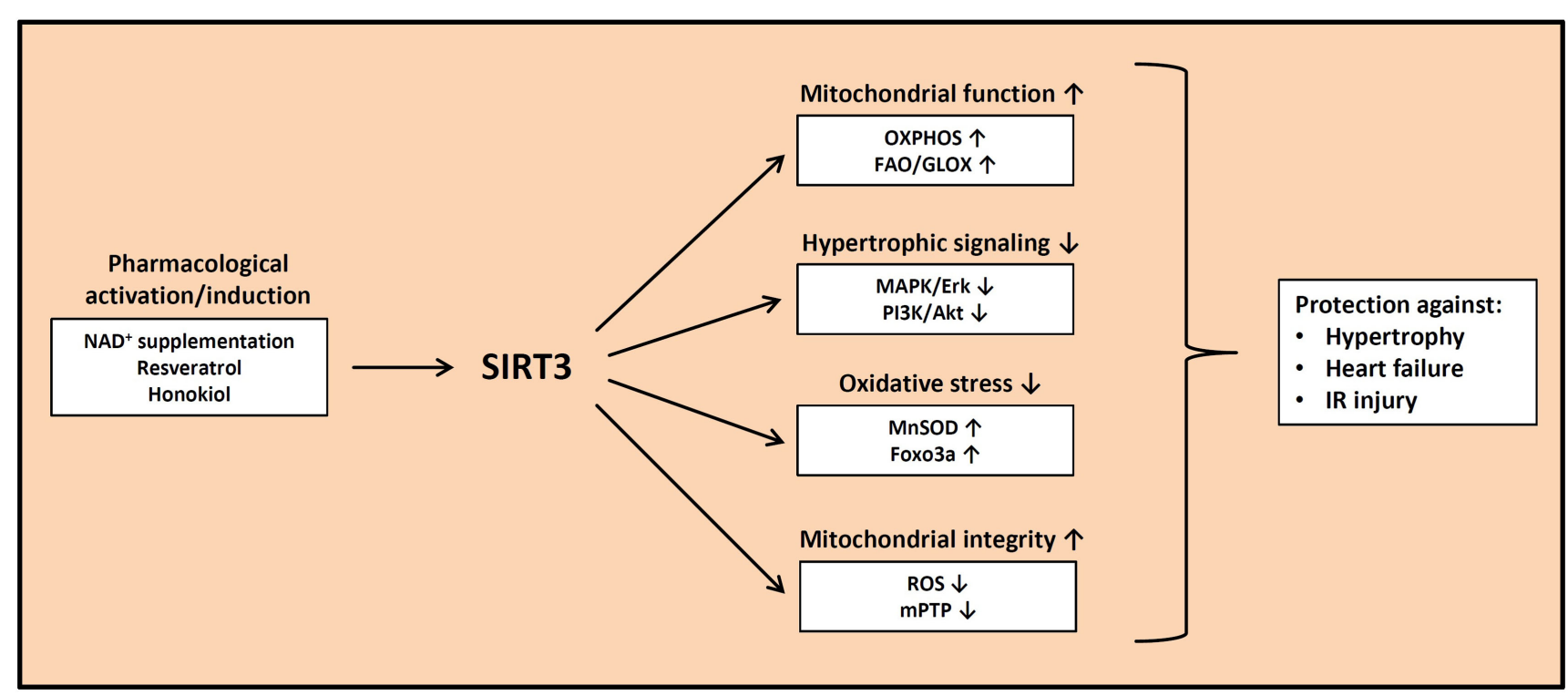

FIGURE 1 | Beneficial effects of increased SIRT3 activity. Pharmacological activation and/or induction of SIRT3 expression may protect against cardiac hypertrophy, heart failure, and ischemia-reperfusion injury by improving mitochondrial substrate oxidation, decreasing prohypertrophic signaling, attenuating oxidative stress, and maintaining mitochondrial integrity. OXPHOS, oxidative phosphorylation; FAO, fatty acid oxidation; GLOX, glucose oxidation; MnSOD, manganese superoxide dismutase; ROS, reactive oxygen species; $\mathrm{MPTP}$, mitochondrial permeability transition pore.

Yet another approach to increase SIRT3 activity is by elevating $\mathrm{NAD}^{+}$levels by feeding the $\mathrm{NAD}^{+}$salvage pathway using the $\mathrm{NAD}^{+}$precursor molecule NMN. By administration of NMN, Pillai et al. could attenuate phenylephrine-induced cardiomyocyte hypertrophy in neonatal rat cardiomyocytes and AngII-induced cardiac hypertrophy in mice (29). Importantly, they also demonstrated that NMN treatment attenuated isoproterenol-induced cardiac hypertrophy, a beneficial effect that was blunted in mice with additional SIRT3 deficiency (29). Even more impressive, Lee et al. recently demonstrated that normalization of the $\mathrm{NAD}^{+}$ redox balance by NMN treatment or overexpression of the ratelimiting enzyme for $\mathrm{NAD}^{+}$synthesis, nicotinamide phosphoribosyltransferase (Nampt), improved cardiac hypertrophy and dysfunction induced by isoproterenol treatment or TAC, strongly suggesting that $\mathrm{NAD}^{+}$repletion may represent a promising therapeutic approach to treat cardiac hypertrophy and heart failure (82). Similar positive effects of $\mathrm{NAD}^{+}$repletion were observed in the setting of IR, where $\mathrm{NAD}^{+}$is lost during reperfusion (63). Reraising $\mathrm{NAD}^{+}$levels by overexpression of Nampt or by administration of NMN resulted in reduced myocardial infarct size following IR $(63,83)$. Although none of the above mentioned substances (honokiol, RSV, NMN) is a specific activator of SIRT3, the results are encouraging that specific activation of SIRT3 as a therapeutic strategy may have beneficial effects in heart disease.

\section{OTHER MITOCHONDRIAL SIRTUINS}

SIRT4 and SIRT5 are further mitochondrial sirtuins that require $\mathrm{NAD}^{+}$as a cosubstrate and which seem to oppose or complement the function of SIRT3 in the heart. Thus far, only few is known about SIRT4 and SIRT5 in general, compared with SIRT3. While the in vitro deacetylation activity of SIRT4 seems to be much lower compared with SIRT3, other posttranslational modifications may be of higher relevance, such as delipoylation and ADP ribosylation, although the latter one may simply represent a side reaction of the main deacylation activity without significant physiological relevance (84-87). SIRT4 has been shown to regulate energy metabolism by inhibiting glutamate dehydrogenase by ADP ribosylation, to inhibit the pyruvate dehydrogenase complex by hydrolyzing the lipoamide cofactors from the E2 component dihydrolipoyllysine acetyltransferase, and to regulate lipid metabolism by deacetylating malonyl-CoA decarboxylase, resulting in malonyl-CoA accumulation and inhibition of FAO (85-87). SIRT4 is highly expressed in the heart, but does not seem to affect the physiologic pump function of the heart when genetically overexpressed or deleted (88). In contrast, increased cardiac hypertrophy and aggravated cardiac dysfunction have been observed in response to AngII treatment in mice with cardiomyocyte-restricted overexpression of SIRT4 (88). Mechanistically, mitochondria-targeted antioxidant treatment prevented the aggravation of cardiac hypertrophy in SIRT4-overexpressing mice, suggesting increased mitochondrial oxidative stress as the cause. The authors proposed that SIRT4 may inhibit binding of SIRT3 to MnSOD, thereby increasing MnSOD acetylation and impairing $\mathrm{H}_{2} \mathrm{O}_{2}$ detoxification (88). Regarding IR injury in the heart, hypoxia downregulated the expression of SIRT4 in H9C2 rat heart myoblasts, and knockdown of SIRT4 decreased cell viability, increased caspase activation, and increased the amount of apoptotic cells, suggesting that downregulation of SIRT4 during hypoxia may contribute to increased apoptosis in response to hypoxia and IR (89). In contrast, SIRT4 siRNA treatment protected against induction of $\mathrm{mPTP}$ opening and mPTP-dependent 
ROS production in another study, leaving the role of SIRT4 in myocardial IR injury incompletely elucidated (90).

SIRT5 also exerts only weak deacetylase activity, but instead catalyzes desuccinylation, demalonylation, and deglutarylation (91, 92). Large-scale proteomic analyses identified many proteins to be putative targets of SIRT5, including enzymes of amino acid degradation, the TCA cycle, FAO, pyruvate decarboxylation, and ATP synthesis (91). SIRT5 is highly expressed in cardiac tissue. Mice with deletion of SIRT5 develop cardiac dysfunction, pathologic hypertrophy, and increased fibrosis (93). Analysis of the cardiac succinylome in SIRT5 ${ }^{-1-}$ mice suggested mitochondrial energy metabolic pathways to be regulated by SIRT5, and that myocardial energy depletion may contribute to cardiac dysfunction in SIRT5 ${ }^{-/-}$mice, potentially due in part to hypersuccinylation and inhibition of the FAO enzyme enoylCoA hydratase $\alpha$ (93). Lack of SIRT5 also modulates the extent of IR injury. Myocardial infarct size was increased, and recovery of contractile function was impaired in hearts of SIRT5 ${ }^{-/}$mice following IR (94). IR injury was restored to WT levels by inhibition of succinate dehydrogenase, which was hypersuccinylated and probably more active in SIRT5 ${ }^{-/-}$hearts. Since succinate accumulates during ischemia and drives mitochondrial ROS generation at the onset of reperfusion by increasing reverse electron transport in the respiratory chain, the authors proposed that inhibition of succinate dehydrogenase may restore IR injury to WT levels by reducing succinate-driven ROS production $(94,95)$.

Taken together, both SIRT4 and SIRT5 may play a role in cardiac pathologies, and more studies are needed to further understand their mechanism of action. Deciphering the role of each mitochondrial sirtuin is important since some of the promising therapeutic approaches, in particular $\mathrm{NAD}^{+}$repletion, should increase the activity of all mitochondrial sirtuins simultaneously.

\section{REFERENCES}

1. Kaeberlein M, McVey M, Guarente L. The SIR2/3/4 complex and SIR2 alone promote longevity in Saccharomyces cerevisiae by two different mechanisms. Genes Dev (1999) 13(19):2570-80. doi:10.1101/gad.13.19.2570

2. Zhang J, Sprung R, Pei J, Tan X, Kim S, Zhu H, et al. Lysine acetylation is a highly abundant and evolutionarily conserved modification in Escherichia coli. Mol Cell Proteomics (2009) 8(2):215-25. doi:10.1074/mcp.M800187-MCP200

3. North BJ, Verdin E. Sirtuins: Sir2-related NAD-dependent protein deacetylases. Genome Biol (2004) 5(5):224. doi:10.1186/gb-2004-5-5-224

4. Anderson RM, Bitterman KJ, Wood JG, Medvedik O, Cohen H, Lin SS, et al. Manipulation of a nuclear NAD+ salvage pathway delays aging without altering steady-state NAD+ levels. JBiol Chem (2002) 277(21):18881-90. doi:10.1074/jbc.M111773200

5. Karamanlidis G, Lee CF, Garcia-Menendez L, Kolwicz SC Jr, Suthammarak W, Gong G, et al. Mitochondrial complex I deficiency increases protein acetylation and accelerates heart failure. Cell Metab (2013) 18(2):239-50. doi:10.1016/j.cmet.2013.07.002

6. Lin SJ, Defossez PA, Guarente L. Requirement of NAD and SIR2 for life-span extension by calorie restriction in Saccharomyces cerevisiae. Science (2000) 289(5487):2126-8. doi:10.1126/science.289.5487.2126

7. Lombard DB, Alt FW, Cheng HL, Bunkenborg J, Streeper RS, Mostoslavsky R, et al. Mammalian Sir2 homolog SIRT3 regulates global mitochondrial lysine acetylation. Mol Cell Biol (2007) 27(24):8807-14. doi:10.1128/MCB.01636-07

8. Bugger H, Witt CN, Bode C. Mitochondrial sirtuins in the heart. Heart Fail Rev (2016) 21(5):519. doi:10.1007/s10741-016-9570-7

\section{CONCLUSION}

Mitochondrial damage and dysfunction contribute to the pathogenesis of different cardiac pathologies, including heart failure and IR injury $(30,31,62)$. Impairment in SIRT3 expression and/or activity has been proposed to contribute to these mitochondrial defects by slowing metabolic substrate oxidation, boostering mitochondrial oxidative stress, and increasing the sensitivity for mPTP induction. Based on beneficial effects on cardiac hypertrophy and IR injury by treatment with honokiol, resveratrol, and $\mathrm{NMN}$, which increase the expression and/or activity of SIRT3, the development of specific SIRT3 activators could represent a novel therapeutic strategy by which multiple mitochondrial defects may be treated simultaneously. It needs to be kept in mind though that SIRT3 may interact with other mitochondrial sirtuins (SIRT4, SIRT5) as well; the myocardial functions of which are still poorly understood. Thus, more studies are needed to deepen our understanding of all mitochondrial sirtuins in cardiac physiology and disease. Based on our current knowledge, modulation of mitochondrial sirtuin activity seems to have great therapeutic potential and may advance the field of mitochondrial medicine also for diseases beyond cardiovascular disease.

\section{AUTHOR CONTRIBUTIONS}

CK wrote the manuscript. $\mathrm{CB}$ and $\mathrm{HB}$ reviewed and edited the manuscript.

\section{FUNDING}

This work was funded by the German Research Foundation (DFG; Bu2126/2-1).

9. Kim SC, Sprung R, Chen Y, Xu Y, Ball H, Pei J, et al. Substrate and functional diversity of lysine acetylation revealed by a proteomics survey. Mol Cell (2006) 23(4):607-18. doi:10.1016/j.molcel.2006.06.026

10. Hebert AS, Dittenhafer-Reed KE, Yu W, Bailey DJ, Selen ES, Boersma MD, et al. Calorie restriction and SIRT3 trigger global reprogramming of the mitochondrial protein acetylome. Mol Cell (2013) 49(1):186-99. doi:10.1016/ j.molcel.2012.10.024

11. Hallows WC, Lee S, Denu JM. Sirtuins deacetylate and activate mammalian acetyl-CoA synthetases. Proc Natl Acad Sci U S A (2006) 103(27):10230-5. doi:10.1073/pnas.0604392103

12. Fernandes J, Weddle A, Kinter CS, Humphries KM, Mather T, Szweda LI, et al. Lysine acetylation activates mitochondrial aconitase in the heart. Biochemistry (2015) 54(25):4008-18. doi:10.1021/acs.biochem.5b00375

13. Hafner AV, Dai J, Gomes AP, Xiao CY, Palmeira CM, Rosenzweig A, et al. Regulation of the MPTP by SIRT3-mediated deacetylation of CypD at lysine 166 suppresses age-related cardiac hypertrophy. Aging (Albany NY) (2010) 2(12):914-23. doi:10.18632/aging.100252

14. Schlicker C, Gertz M, Papatheodorou P, Kachholz B, Becker CF, Steegborn C. Substrates and regulation mechanisms for the human mitochondrial sirtuins Sirt3 and Sirt5.J Mol Biol (2008) 382(3):790-801.doi:10.1016/j.jmb.2008.07.048

15. Sundaresan NR, Samant SA, Pillai VB, Rajamohan SB, Gupta MP. SIRT3 is a stress-responsive deacetylase in cardiomyocytes that protects cells from stress-mediated cell death by deacetylation of Ku70. Mol Cell Biol (2008) 28(20):6384-401. doi:10.1128/MCB.00426-08

16. Hirschey MD, Shimazu T, Goetzman E, Jing E, Schwer B, Lombard DB, et al. SIRT3 regulates mitochondrial fatty-acid oxidation by reversible 
enzyme deacetylation. Nature (2010) 464(7285):121-5. doi:10.1038/nature 08778

17. Sundaresan NR, Gupta M, Kim G, Rajamohan SB, Isbatan A, Gupta MP. Sirt3 blocks the cardiac hypertrophic response by augmenting Foxo3a-dependent antioxidant defense mechanisms in mice. J Clin Invest (2009) 119(9):2758-71. doi:10.1172/JCI39162

18. Mori J, Alrob OA, Wagg CS, Harris RA, Lopaschuk GD, Oudit GY. ANG II causes insulin resistance and induces cardiac metabolic switch and inefficiency: a critical role of PDK4. Am J Physiol Heart Circ Physiol (2013) 304(8):H1103-13. doi:10.1152/ajpheart.00636.2012

19. Cimen H, Han MJ, Yang Y, Tong Q, Koc H, Koc EC. Regulation of succinate dehydrogenase activity by SIRT3 in mammalian mitochondria. Biochemistry (2010) 49(2):304-11. doi:10.1021/bi901627u

20. Finley LW, Haas W, Desquiret-Dumas V, Wallace DC, Procaccio V, Gygi SP, et al. Succinate dehydrogenase is a direct target of sirtuin 3 deacetylase activity. PLoS One (2011) 6(8):e23295. doi:10.1371/journal.pone.0023295

21. Bharathi SS, Zhang Y, Mohsen AW, Uppala R, Balasubramani M, Schreiber E, et al. Sirtuin 3 (SIRT3) protein regulates long-chain acyl-CoA dehydrogenase by deacetylating conserved lysines near the active site. J Biol Chem (2013) 288(47):33837-47. doi:10.1074/jbc.M113.510354

22. Koentges C, Pfeil K, Meyer-Steenbuck M, Lother A, Hoffmann MM, Odening KE, et al. Preserved recovery of cardiac function following ischemia-reperfusion in mice lacking SIRT3. Can J Physiol Pharmacol (2016) 94(1):72-80. doi:10.1139/cjpp-2015-0152

23. Koentges C, Pfeil K, Schnick T, Wiese S, Dahlbock R, Cimolai MC, et al. SIRT3 deficiency impairs mitochondrial and contractile function in the heart. Basic Res Cardiol (2015) 110(4):36. doi:10.1007/s00395-015-0493-6

24. Alrob OA, Sankaralingam S, Ma C, Wagg CS, Fillmore N, Jaswal JS, et al. Obesity-induced lysine acetylation increases cardiac fatty acid oxidation and impairs insulin signalling. Cardiovasc Res (2014) 103(4):485-97. doi:10.1093/ $\mathrm{cvr} / \mathrm{cvu} 156$

25. Chen T, Liu J, Li N, Wang S, Liu H, Li J, et al. Mouse SIRT3 attenuates hypertrophy-related lipid accumulation in the heart through the deacetylation of LCAD. PLoS One (2015) 10(3):e0118909. doi:10.1371/journal.pone.0118909

26. Porter GA, Urciuoli WR, Brookes PS, Nadtochiy SM. SIRT3 deficiency exacerbates ischemia-reperfusion injury: implication for aged hearts. Am J Physiol Heart Circ Physiol (2014) 306(12):H1602-9. doi:10.1152/ajpheart.00027.2014

27. Cheung KG, Cole LK, Xiang B, Chen K, Ma X, Myal Y, et al. Sirtuin-3 (SIRT3) protein attenuates doxorubicin-induced oxidative stress and improves mitochondrial respiration in H9c2 cardiomyocytes. J Biol Chem (2015) 290(17):10981-93. doi:10.1074/jbc.M114.607960

28. Zeng H, Vaka VR, He X, Booz GW, Chen JX. High-fat diet induces cardiac remodelling and dysfunction: assessment of the role played by SIRT3 loss. J Cell Mol Med (2015) 19(8):1847-56. doi:10.1111/jcmm.12556

29. Pillai VB, Sundaresan NR, Kim G, Gupta M, Rajamohan SB, Pillai JB, et al. Exogenous NAD blocks cardiac hypertrophic response via activation of the SIRT3-LKB1-AMP-activated kinase pathway. J Biol Chem (2010) 285(5):3133-44. doi:10.1074/jbc.M109.077271

30. Stanley WC, Recchia FA, Lopaschuk GD. Myocardial substrate metabolism in the normal and failing heart. Physiol Rev (2005) 85(3):1093-129. doi:10.1152/ physrev.00006.2004

31. Neubauer S. The failing heart - an engine out of fuel. N Engl J Med (2007) 356(11):1140-51. doi:10.1056/NEJMra063052

32. Halestrap AP, Clarke SJ, Khaliulin I. The role of mitochondria in protection of the heart by preconditioning. Biochim Biophys Acta (2007) 1767(8):1007-31. doi:10.1016/j.bbabio.2007.05.008

33. Halestrap AP, Clarke SJ, Javadov SA. Mitochondrial permeability transition pore opening during myocardial reperfusion - a target for cardioprotection. Cardiovasc Res (2004) 61(3):372-85. doi:10.1016/S0008-6363(03)00533-9

34. Schwarz K, Siddiqi N, Singh S, Neil CJ, Dawson DK, Frenneaux MP. The breathing heart - mitochondrial respiratory chain dysfunction in cardiac disease. Int J Cardiol (2014) 171(2):134-43. doi:10.1016/j.ijcard.2013.12.014

35. Stride N, Larsen S, Hey-Mogensen M, Sander K, Lund JT, Gustafsson F, et al. Decreased mitochondrial oxidative phosphorylation capacity in the human heart with left ventricular systolic dysfunction. Eur J Heart Fail (2013) 15(2):150-7. doi:10.1093/eurjhf/hfs172

36. Tsutsui H, Kinugawa $S$, Matsushima S. Oxidative stress and heart failure. Am JPhysiol Heart Circ Physiol (2011) 301(6):H2181-90. doi:10.1152/ ajpheart.00554.2011
37. Zeng L, Yang Y, Hu Y, Sun Y, Du Z, Xie Z, et al. Age-related decrease in the mitochondrial sirtuin deacetylase Sirt3 expression associated with ROS accumulation in the auditory cortex of the mimetic aging rat model. PLoS One (2014) 9(2):e88019. doi:10.1371/journal.pone.0088019

38. Martin-Fernandez B, Gredilla R. Mitochondria and oxidative stress in heart aging. Age (Dordr) (2016). doi:10.1007/s11357-016-9933-y

39. Pillai VB, Samant S, Sundaresan NR, Raghuraman H, Kim G, Bonner MY, et al. Honokiol blocks and reverses cardiac hypertrophy in mice by activating mitochondrial Sirt3. Nat Commun (2015) 6:6656. doi:10.1038/ncomms7656

40. Ahn BH, Kim HS, Song S, Lee IH, Liu J, Vassilopoulos A, et al. A role for the mitochondrial deacetylase Sirt3 in regulating energy homeostasis. Proc Natl Acad Sci U S A (2008) 105(38):14447-52. doi:10.1073/pnas.0803790105

41. Liang L, Li Q, Huang L, Li D, Li X. Sirt3 binds to and deacetylates mitochondrial pyruvate carrier 1 to enhance its activity. Biochem Biophys Res Commun (2015) 468(4):807-12. doi:10.1016/j.bbrc.2015.11.036

42. Sahar S, Nin V, Barbosa MT, Chini EN, Sassone-Corsi P. Altered behavioral and metabolic circadian rhythms in mice with disrupted NAD+ oscillation. Aging (Albany NY) (2011) 3(8):794-802. doi:10.18632/aging.100368

43. Tao R, Coleman MC, Pennington JD, Ozden O, Park SH, Jiang H, et al. Sirt3-mediated deacetylation of evolutionarily conserved lysine 122 regulates MnSOD activity in response to stress. Mol Cell (2010) 40(6):893-904. doi:10.1016/j.molcel.2010.12.013

44. Qiu X, Brown K, Hirschey MD, Verdin E, Chen D. Calorie restriction reduces oxidative stress by SIRT3-mediated SOD2 activation. Cell Metab (2010) 12(6):662-7. doi:10.1016/j.cmet.2010.11.015

45. Brooks CL, Gu W. The impact of acetylation and deacetylation on the p53 pathway. Protein Cell (2011) 2(6):456-62. doi:10.1007/s13238-011-1063-9

46. Bao J, Scott I, Lu Z, Pang L, Dimond CC, Gius D, et al. SIRT3 is regulated by nutrient excess and modulates hepatic susceptibility to lipotoxicity. Free Radic Biol Med (2010) 49(7):1230-7. doi:10.1016/j.freeradbiomed.2010.07.009

47. Pillai VB, Bindu S, Sharp W, Fang YH, Kim G, Gupta M, et al. Sirt3 protects mitochondrial DNA damage and blocks the development of doxorubicin-induced cardiomyopathy in mice. Am J Physiol Heart Circ Physiol (2016) 310(8):H962-72. doi:10.1152/ajpheart.00832.2015

48. Grillon JM, Johnson KR, Kotlo K, Danziger RS. Non-histone lysine acetylated proteins in heart failure. Biochim Biophys Acta (2012) 1822(4):607-14. doi:10.1016/j.bbadis.2011.11.016

49. Parodi-Rullan R, Barreto-Torres G, Ruiz L, Casasnovas J, Javadov S. Direct renin inhibition exerts an anti-hypertrophic effect associated with improved mitochondrial function in post-infarction heart failure in diabetic rats. Cell Physiol Biochem (2012) 29(5-6):841-50. doi:10.1159/000178526

50. Zeng H, He X, Hou X, Li L, Chen JX. Apelin gene therapy increases myocardial vascular density and ameliorates diabetic cardiomyopathy via upregulation of sirtuin 3. Am J Physiol Heart Circ Physiol (2014) 306(4):H585-97. doi:10.1152/ ajpheart.00821.2013

51. Hou X, Zeng H, He X, Chen JX. Sirt3 is essential for apelin-induced angiogenesis in post-myocardial infarction of diabetes. J Cell Mol Med (2015) 19(1):53-61. doi:10.1111/jcmm.12453

52. Vazquez EJ, Berthiaume JM, Kamath V, Achike O, Buchanan E, Montano $\mathrm{MM}$, et al. Mitochondrial complex I defect and increased fatty acid oxidation enhance protein lysine acetylation in the diabetic heart. Cardiovasc Res (2015) 107(4):453-65. doi:10.1093/cvr/cvv183

53. Sultana MR, Bagul PK, Katare PB, Anwar S, Padiya R, Banerjee SK. Garlic activates SIRT- 3 to prevent cardiac oxidative stress and mitochondrial dysfunction in diabetes. Life Sci (2016) 164:42-51. doi:10.1016/j.lfs.2016.08.030

54. Kong X, Wang R, Xue Y, Liu X, Zhang H, Chen Y, et al. Sirtuin 3, a new target of PGC-1alpha, plays an important role in the suppression of ROS and mitochondrial biogenesis. PLoS One (2010) 5(7):e11707. doi:10.1371/journal. pone. 0011707

55. Shi L, Zhang T, Zhou Y, Zeng X, Ran L, Zhang Q, et al. Dihydromyricetin improves skeletal muscle insulin sensitivity by inducing autophagy via the AMPK-PGC-1alpha-Sirt3 signaling pathway. Endocrine (2015) 50(2):378-89. doi:10.1007/s12020-015-0599-5

56. Bugger H, Abel ED. Mitochondria in the diabetic heart. Cardiovasc Res (2010) 88(2):229-40. doi:10.1093/cvr/cvq239

57. Tao L, Bei Y, Zhang H, Xiao J, Li X. Exercise for the heart: signaling pathways. Oncotarget (2015) 6(25):20773-84. doi:10.18632/oncotarget.4770

58. Sundaresan NR, Bindu S, Pillai VB, Samant S, Pan Y, Huang JY, et al. SIRT3 blocks aging-associated tissue fibrosis in mice by deacetylating and 
activating glycogen synthase kinase 3beta. Mol Cell Biol (2015) 36(5):678-92. doi:10.1128/MCB.00586-15

59. Pacher P, Szabo C. Role of poly(ADP-ribose) polymerase 1 (PARP-1) in cardiovascular diseases: the therapeutic potential of PARP inhibitors. Cardiovasc Drug Rev (2007) 25(3):235-60. doi:10.1111/j.1527-3466.2007.00018.x

60. Yang $H$, Yang T, Baur JA, Perez E, Matsui T, Carmona JJ, et al. Nutrient-sensitive mitochondrial NAD+ levels dictate cell survival. Cell (2007) 130(6):1095-107. doi:10.1016/j.cell.2007.07.035

61. Yellon DM, Hausenloy DJ. Myocardial reperfusion injury. N Engl J Med (2007) 357(11):1121-35. doi:10.1056/NEJMra071667

62. Hausenloy DJ, Yellon DM. Myocardial ischemia-reperfusion injury: a neglected therapeutic target. J Clin Invest (2013) 123(1):92-100. doi:10.1172/ JCI62874

63. Di Lisa F, Menabo R, Canton M, Barile M, Bernardi P. Opening of the mitochondrial permeability transition pore causes depletion of mitochondrial and cytosolic NAD+ and is a causative event in the death of myocytes in postischemic reperfusion of the heart. J Biol Chem (2001) 276(4):2571-5. doi:10.1074/jbc.M006825200

64. Korzick DH, Lancaster TS. Age-related differences in cardiac ischemia-reperfusion injury: effects of estrogen deficiency. Pflugers Arch (2013) 465(5):66985. doi:10.1007/s00424-013-1255-7

65. Lesnefsky EJ, Gallo DS, Ye J, Whittingham TS, Lust WD. Aging increases ischemia-reperfusion injury in the isolated, buffer-perfused heart. J Lab Clin Med (1994) 124(6):843-51.

66. Fannin SW, Lesnefsky EJ, Slabe TJ, Hassan MO, Hoppel CL. Aging selectively decreases oxidative capacity in rat heart interfibrillar mitochondria. Arch Biochem Biophys (1999) 372(2):399-407. doi:10.1006/abbi.1999.1508

67. Judge S, Jang YM, Smith A, Hagen T, Leeuwenburgh C. Age-associated increases in oxidative stress and antioxidant enzyme activities in cardiac interfibrillar mitochondria: implications for the mitochondrial theory of aging. FASEB J (2005) 19(3):419-21. doi:10.1096/fj.04-2622fje

68. Karbowski M, Kurono C, Wozniak M, Ostrowski M, Teranishi M, Nishizawa $\mathrm{Y}$, et al. Free radical-induced megamitochondria formation and apoptosis. Free Radic Biol Med (1999) 26(3-4):396-409. doi:10.1016/S0891-5849(98)00209-3

69. Sachs HG, Colgan JA, Lazarus ML. Ultrastructure of the aging myocardium: a morphometric approach. Am J Anat (1977) 150(1):63-71. doi:10.1002/ aja. 1001500105

70. Sohal RS, Dubey A. Mitochondrial oxidative damage, hydrogen peroxide release, and aging. Free Radic Biol Med (1994) 16(5):621-6. doi:10.1016/0891-5849(94)90062-0

71. Sohal RS, Ku HH, Agarwal S, Forster MJ, Lal H. Oxidative damage, mitochondrial oxidant generation and antioxidant defenses during aging and in response to food restriction in the mouse. Mech Ageing Dev (1994) 74(1-2):121-33. doi:10.1016/0047-6374(94)90104-X

72. Wang XQ, Shao Y, Ma CY, Chen W, Sun L, Liu W, et al. Decreased SIRT3 in aged human mesenchymal stromal/stem cells increases cellular susceptibility to oxidative stress. JCell Mol Med (2014) 18(11):2298-310. doi:10.1111/ jcmm. 12395

73. Kwon Y, Kim J, Lee CY, Kim H. Expression of SIRT1 and SIRT3 varies according to age in mice. Anat Cell Biol (2015) 48(1):54-61. doi:10.5115/ acb.2015.48.1.54

74. Gomes AP, Price NL, Ling AJ, Moslehi JJ, Montgomery MK, Rajman L, et al. Declining $\mathrm{NAD}(+)$ induces a pseudohypoxic state disrupting nuclear-mitochondrial communication during aging. Cell (2013) 155(7):1624-38. doi:10.1016/j.cell.2013.11.037

75. Mouchiroud L, Houtkooper RH, Moullan N, Katsyuba E, Ryu D, Canto C, et al. The $\mathrm{NAD}(+) /$ sirtuin pathway modulates longevity through activation of mitochondrial UPR and FOXO signaling. Cell (2013) 154(2):430-41. doi:10.1016/j.cell.2013.06.016

76. Yoshino J, Mills KF, Yoon MJ, Imai S. Nicotinamide mononucleotide, a key $\mathrm{NAD}(+)$ intermediate, treats the pathophysiology of diet- and age-induced diabetes in mice. Cell Metab (2011) 14(4):528-36. doi:10.1016/ j.cmet.2011.08.014

77. Averett C, Arora S, Zubair H, Singh S, Bhardwaj A, Singh AP. Molecular targets of Honokiol: a promising phytochemical for effective cancer management. Enzymes (2014) 36:175-93. doi:10.1016/B978-0-12-802215-3.00009-4

78. Kumar A, Kumar Singh U, Chaudhary A. Honokiol analogs: a novel class of anticancer agents targeting cell signaling pathways and other bioactivities. Future Med Chem (2013) 5(7):809-29. doi:10.4155/fmc.13.32
79. Zordoky BN, Robertson IM, Dyck JR. Preclinical and clinical evidence for the role of resveratrol in the treatment of cardiovascular diseases. Biochim Biophys Acta (2015) 1852(6):1155-77. doi:10.1016/j.bbadis.2014.10.016

80. Han G, Xia J, Gao J, Inagaki Y, Tang W, Kokudo N. Anti-tumor effects and cellular mechanisms of resveratrol. Drug Discov Ther (2015) 9(1):1-12. doi:10.5582/ddt.2015.01007

81. Chen T, Li J, Liu J, Li N, Wang S, Liu H, et al. Activation of SIRT3 by resveratrol ameliorates cardiac fibrosis and improves cardiac function via the TGF-beta/ Smad3 pathway. Am J Physiol Heart Circ Physiol (2015) 308(5):H424-34. doi:10.1152/ajpheart.00454.2014

82. Lee CF, Chavez JD, Garcia-Menendez L, Choi Y, Roe ND, Chiao YA, et al. Normalization of NAD+ redox balance as a therapy for heart failure. Circulation (2016) 134(12):883-94. doi:10.1161/CIRCULATIONAHA.116.022495

83. Yamamoto T, Byun J, Zhai P, Ikeda Y, Oka S, Sadoshima J. Nicotinamide mononucleotide, an intermediate of NAD+ synthesis, protects the heart from ischemia and reperfusion. PLoS One (2014) 9(6):e98972. doi:10.1371/journal. pone. 0098972

84. Gertz M, Steegborn C. Using mitochondrial sirtuins as drug targets: disease implications and available compounds. Cell Mol Life Sci (2016) 73(15):287196. doi:10.1007/s00018-016-2180-7

85. Haigis MC, Mostoslavsky R, Haigis KM, Fahie K, Christodoulou DC, Murphy AJ, et al. SIRT4 inhibits glutamate dehydrogenase and opposes the effects of calorie restriction in pancreatic beta cells. Cell (2006) 126(5):941-54. doi:10.1016/j.cell.2006.06.057

86. Laurent G, German NJ, Saha AK, de Boer VC, Davies M, Koves TR, et al. SIRT4 coordinates the balance between lipid synthesis and catabolism by repressing malonyl CoA decarboxylase. Mol Cell (2013) 50(5):686-98. doi:10.1016/j. molcel.2013.05.012

87. Mathias RA, Greco TM, Oberstein A, Budayeva HG, Chakrabarti R, Rowland EA, et al. Sirtuin 4 is a lipoamidase regulating pyruvate dehydrogenase complex activity. Cell (2014) 159(7):1615-25. doi:10.1016/j.cell.2014. 11.046

88. Luo YX, Tang X, An XZ, Xie XM, Chen XF, Zhao X, et al. Sirt4 accelerates Ang II-induced pathological cardiac hypertrophy by inhibiting manganese superoxide dismutase activity. Eur Heart J (2016). doi:10.1093/eurheartj/ehw138

89. Liu B, Che W, Xue J, Zheng C, Tang K, Zhang J, et al. SIRT4 prevents hypoxia-induced apoptosis in H9c2 cardiomyoblast cells. Cell Physiol Biochem (2013) 32(3):655-62. doi:10.1159/000354469

90. Verma M, Shulga N, Pastorino JG. Sirtuin-4 modulates sensitivity to induction of the mitochondrial permeability transition pore. Biochim Biophys Acta (2013) 1827(1):38-49. doi:10.1016/j.bbabio.2012.09.016

91. Rardin MJ, He W, Nishida Y, Newman JC, Carrico C, Danielson SR, et al. SIRT5 regulates the mitochondrial lysine succinylome and metabolic networks. Cell Metab (2013) 18(6):920-33. doi:10.1016/j.cmet.2013.11.013

92. Peng C, Lu Z, Xie Z, Cheng Z, Chen Y, Tan M, et al. The first identification of lysine malonylation substrates and its regulatory enzyme. Mol Cell Proteomics (2011) 10(12):M111012658. doi:10.1074/mcp.M111.012658

93. Sadhukhan S, Liu X, Ryu D, Nelson OD, Stupinski JA, Li Z, et al. Metabolomicsassisted proteomics identifies succinylation and SIRT5 as important regulators of cardiac function. Proc Natl Acad Sci U S A (2016) 113(16):4320-5. doi:10.1073/pnas.1519858113

94. Boylston JA, Sun J, Chen Y, Gucek M, Sack MN, Murphy E. Characterization of the cardiac succinylome and its role in ischemia-reperfusion injury. $J \mathrm{Mol}$ Cell Cardiol (2015) 88:73-81. doi:10.1016/j.yjmcc.2015.09.005

95. Chouchani ET, Pell VR, Gaude E, Aksentijevic D, Sundier SY, Robb EL, et al. Ischaemic accumulation of succinate controls reperfusion injury through mitochondrial ROS. Nature (2014) 515(7527):431-5. doi:10.1038/ nature13909

Conflict of Interest Statement: The authors declare that the research was conducted in the absence of any commercial or financial relationships that could be construed as a potential conflict of interest.

Copyright $\odot 2016$ Koentges, Bode and Bugger. This is an open-access article distributed under the terms of the Creative Commons Attribution License (CC BY). The use, distribution or reproduction in other forums is permitted, provided the original author(s) or licensor are credited and that the original publication in this journal is cited, in accordance with accepted academic practice. No use, distribution or reproduction is permitted which does not comply with these terms. 